\title{
VERY HIGH MORTALITY OF CARDIOMETABOLIC DISEASE IN COVID-19 AND NON-COVID-19 POPULATION DURING THE PANDEMIC FIRST WAVE IN PERU
}

\section{Segundo Nicolas Seclen ${ }^{1}$, Marlon Yovera-Aldana ${ }^{2}$}

${ }^{1}$ Unidad de Diabetes, Hipertensión y Lipidos, Instituto de Gerontologia, Universidad Peruana Cayetano Heredia, Lima, Perú

${ }^{2}$ Grupo de Investigación en Neurociencias, Efectividad Clínica y Salud Pública, Universidad Científica del Sur, Lima, Perú

Corresponding Author: Segundo Nicolas Seclen

Email: segundo.seclen@upch.pe

\section{ABSTRACT}

OBJECTIVE: To describe the mortality of cardio-metabolic diseases by non-COVID-19 during the pandemic first wave 2020 .

METHODOLOGY: We analyzed open data from Peruvian death certificates from March 01 to December 31, 2020, and 2019. Confirmed and suspected cases of COVID-19 by ICD-10 and the text of the diagnosis were included. We calculate the excess mortality 2020/2019 according to demographic characteristics, cardiometabolic disease, a primary cause of death. We performed a Poisson regression analysis for the prevalence rates of non-communicable 
diseases non-COVID-19 / COVID-19 adjusted to sex, age, place of deaths, Insurance, altitude, poverty quintile, precedence regio, degree of instruction.

RESULTS: Deaths registered in first wave compared to 2019 resulted excess of 92,395 (+ $96 \%$ ). The mortality rate was $25.1 / 10,000$ inhabitants, $60 \%$ were male, $72.6 \%$ were older than 60 years and $38 \%$ died in Lima/Callao. $57 \%$ of deaths were due non-COVID disease. In this population, stroke was 51\% more frequent (PR 1.51; CI95\% 1.49-1.52; p $<0.001$ ); coronary disease was 29\% (PR 1.29 CI95\% 1.28-1.30;p<0.001), an diabetes was 5\% (PR 1.06; CI95\% 1.05-1.08; $<0.001$ ). In COVID subjects, only obesity was more frequent in 37\% (PR 1.37; CI95\%1.35-1.-39; $<0.001$ ). There was an increase in cardiovascular death of $113 \%$ compared to 2019. Likewise, it was 13.9 times more frequent in non-COVID-19 compared to COVID-19 .(PR 13.29; CI95\% 12.32 - 14.34; p<0.001) adjusted a epidemiological characteristics.

CONCLUSION: During the first wave of COVID-19 in Peru, six out of ten were unrelated to COVID-19. The associated cardiometabolic diseases in COVID-19 deaths were obesity, while non-COVID-19 deaths were stroke, coronary disease, and diabetes mellitus.

Keywords: COVID-19; mortality; diabetes mellitus; hypertension; coronary disease; stroke; obesity; cardiovascular diseases.

\section{INTRODUCTION}

The COVID-19 pandemic due to high morbidity and excess mortality has collapsed the world's different health systems, affecting high, middle, and low-income countries [1]. Many deaths have happened in the uninfected population by delays in health care and the inefficiency of health systems, mainly in countries with limited resources. Direct and indirect deaths have been primarily to cardiometabolic diseases as diabetes, hypertension, and coronary heart disease $[2,3]$.

In developed countries with efficient vital registration systems and confirmation tests, the excess of death oscillated between 20-50\%, and only 40-70\% was due COVID19 [4,5]. In developing countries, such as Latin America, information systems have great structural difficulties [6]. Peru is one of the countries with the highest mortality. In the begginnig of first wave, Perú doesn't made an extensive molecular test for shortage of diagnostic tests. This 
delayed the reporting of confirmatory COVID-19 cases, producing inexact information of mortality [7,8]. The excess deaths by COVID-19 and non-COVID-19 are relevant in public health decisions.

Previous to the pandemic, there was an increase in mortality from non-communicable diseases from $63.9 \%$ to $83.6 \%$ between $2003-2016$ in Peru[9]. In a similar period, the death attributed to diabetes mellitus (DM) was $2.7 \%$, and the population mortality in 2015 was $9.5 \%$ [10]. In the actual context, it was predictable higher morbidity and mortality in cardiometabolic diseases.

The database of the National Death Information System (SINADEF in Spanish) collects information from death certificates filled out by physicians. This system is the most accurate database of deaths at the nation-wide [11]. This investigation describes the excess of mortality in the Peruvian population during the first wave of the COVID-19 pandemic, emphasizing cardiometabolic diseases such as diabetes, hypertension, obesity, and cardiovascular disease.

\section{MATERIALS AND METHODS}

\section{Design and study population}

We performed a cross-sectional analysis of the deaths registered in the National System of Deaths of Peru (SINADEF) from March 01 to December 31, 2020. We compared it with the same period from 2019. SINADEF is an open data register of Peruvian deaths since 2017. Only physicians can register online way through its official website https://www.datosabiertos.gob.pe/dataset/informaci\%C3\%B3n-de-fallecidos-del-sistema-

\section{inform $\% \mathrm{C} 3 \% \mathrm{~A} 1$ tico-nacional-de-defunciones-sinadef-ministerio [12]}

Peru is in the central and western parts of South America. They have 33 million inhabitants, and a third of the population is in Lima, its capital. The health system is limited in infrastructure, equipment, and trained personnel, with partial coverage, without developed primary care.

COVID 19 was detected for the first time in Lima on March 06, 2020, and the first death happened on March 19. The peak of fatalities occurred during May to August, culminating the first wave at the end of November, being the lowest point since the beginning of the pandemic, but even higher compared to 2019 
The study included citizens who died in Peru and had Peruvian residence addresses. We exclude subjects without cause of death in the online register.

\section{COVID death}

The deaths with COVID-19 were registered according to the International Classification of Disease (ICD10). The confirmed code was U07.1. Suspect code was U07.2; B34X and B97X. Those without ICD10 code but with the following descriptions: "COVID," "Coronavirus," or "SARS" in the death certificate were included.[13]

\section{Demographic characteristics}

We collected the following information: sex (Female/male), age group $(<18 ; 18-59 ; \geq 60$ years old), instruction degree (illiterate; elementary; high; college, not specified), health insurance (government insurance, formal workers, army force, private, not identified); place of death (health center, home, public/transit road, not specified) altitude $(<500,500-2999,>3000$ above see meters level), natural region (Lima/Callao, coast, highlands, jungle), and monetary poverty quintile ( $1^{\text {st }}$ to $5^{\text {th }}$ quintile, the first quintile was the poorest).

The poverty quintile, altitude, and natural region data of the 1854 Peruvian districts were obtained from Peru's National Institute of Statistics and Informatics. [14-16].

\section{Cardiometabolic diseases and basic death cause.}

Diabetes mellitus, hypertension, obesity, coronary disease, and stroke were defined according to the ICD 10 code record in the death certificate. In patients who did not present coding, we searched a combination of terms according to Supplementary Table 1.

Supplementary table 1. ICD10 codes of cardiometabolic diseases.

\begin{tabular}{|c|c|c|}
\hline & ICD10 & Text word \\
\hline Diabetes Mellitus & E10.0 a E14.9 & "mellit","melit", "diabetic", "hiperglic". \\
\hline Hipertension arterial & I11 a I15 & $\begin{array}{c}\text { "Hipertensión art", "HTA", "HT arterial", } \\
\text { "hipertensiv" }\end{array}$ \\
\hline Obesity & E66 & "Obesidad", "sobrepeso", "exceso de peso". \\
\hline Cardiovascular disease & I20 a I25 & $\begin{array}{c}\text { "IMA", "Infarto mioc", "infarto de m", "infarto } \\
\text { car", "infarto agu", "angina", "coronario", } \\
\text { "SICA" "SCA" }\end{array}$ \\
\hline Brain-vascular disease & I60 a I69 & $\begin{array}{c}\text { "cerebrovascular", "cerebro vascular", "ACV", } \\
\text { "ECV", "DCV", "Ictus", "stroke", "infarto cer", } \\
\text { "A.C.V." }\end{array}$ \\
\hline Kidney disease & N17- N19 & $\begin{array}{c}\text { "enfermedad renal", "ERC", "IRC", "IRCT - } \\
\text { HD", "renal ag", "renal cr".[31] }\end{array}$ \\
\hline
\end{tabular}


The Peruvian death certificates contain three causes of death: direct, intermediate, and basic. The basic cause of death initiates the chain of pathological events that leads directly to death and is used to measure mortality attributed to some diseases.[17,18]. The basic cause of death was regrouped according to a modification of the 10/110 strategy of the Pan American Health Organization [19], according to Supplementary Table 2.

Supplementary table 2. ICD10 codes

\begin{tabular}{|c|c|}
\hline & ICD 10 \\
\hline Respiratory infection. & J09 a J22 \\
\hline Other infectious diseases & A00 -A99, B00-B99, J00-J15, G00-G03 \\
\hline Cardiovascular disease & I20 - I25, I60 a I69 \\
\hline Non- communicable disease & C, D, E, F, G, H, I00 a I19, I26 a I59; I70 a I99, K, L, M, \\
N, O, P, Q y J30 a J99 \\
\hline External injury & S, T, V, W, X, Y \\
\hline Non-specified & "Paro cardio-respiratorio", "Descomposición sin \\
posibilidad de saber causa" \\
"Paro cardiaco"
\end{tabular}

\section{Data analysis}

The SINADEF database was downloaded on January 10, 2021, when COVID-19 first wave finished. In the Microsoft Excel spreadsheet (Microsoft, Redmond, Washington, USA), we evaluated the presence of extreme values, recoded the texts to pre-specified codes, and generated new variables. Stata v 15.1 program (Stata Corp, College Station, Texas, USA) was used to perform statistical analysis with a significance level of $0.05 \%$.

We obtained the percentage of non-reported COVID-19 death of the official Peruvian database of the Ministry of Health (MINSA) [20] compared with the SINADEF database [12].

Excess mortality was calculated between March 01 to December 31th, 2020, and 2019 according to demographic data, cardiometabolic diseases, and basic cause of death.

Using the Pearson chi test, we compared clinical characteristics according to COVID-19 in the same period and evaluated differences between groups. We considered a significance level of $5 \%$.

We calculated the prevalence of general mortality and prevalence rate of each cardiometabolic disease. The prevalence rate was obtained through the Generalized Linear model with the Poisson family, log link and robust variance. We adjusted to sex, age group, 
instruction degree, health insurance, place of death, altitude, and natural region. We focused each general mortality PR in COVID subject and non-COVID subjects with a confidence interval of $95 \%$

Likewise, we calculated the prevalence of cardiovascular death. We calculated adjusted prevalence rates to non- covid disease, sex, age group, instruction degree, health insurance, place of death, altitude, and natural region in the same way.

\section{RESULTS}

\section{Excess of deaths}

Between March 01, 2020, and December 31th 2020, Peru experienced deaths, 96\% more than expected. In general, basic cause of death during the pandemic was mainly respiratory infectious with an excess of $194 \%$. While cardiovascular death was $113 \%$ compared with 2019. All cardio-metabolic diseases had an excess of death, mainly DM, coronary heart disease, HTN, and obesity, with $176 \%, 192 \%, 205 \%$, and $1365 \%$, respectively, compared to 2019. (Table1)

Table 1. Excess mortality according to the socio-demographic characteristic of deaths in $2019-2020$

\begin{tabular}{lccccccc} 
& \multicolumn{2}{c}{ Deaths } & \multicolumn{2}{c}{ Deaths } & \multicolumn{2}{c}{ Mortality } \\
& \multicolumn{2}{c}{2019} & \multicolumn{2}{c}{2020} & \multicolumn{2}{c}{ Excess } \\
& $\mathrm{n}$ & $(\%)$ & $\mathrm{n}$ & $\%$ & $\Delta$ & $\%$ \\
\hline General & 96083 & $(100)$ & 188478 & $(100)$ & 92395 & $(96)$
\end{tabular}

Sex

$\begin{array}{lcccccc}\text { Female } & 44346 & (46.2) & 74189 & (39.4) & 29843 & (67) \\ \text { Male } & 51730 & (53.8 & 114285 & (60.6) & 62555 & (121)\end{array}$

Age group

$\begin{array}{lccccc}<18 \text { y } & 6963 & (7.3) & 5810 & (3.1) & -1153 \\ 18-59 y & 22878 & (23.8) & 45804 & (24.3) & 22926 \\ >60 y & 66182 & (68.9) & 136812 & (72.6) & 70630\end{array}$

\section{Instruction degree}

Illiterate

Elementary School

High School

$\begin{array}{lllll}16577 & (17.3) & 25006 & (13.3) & 8429 \\ 28369 & (29.5) & 44339 & (23.5) & 15970 \\ 20397 & (21.2) & 42889 & (22.8) & 22492\end{array}$


College/Technical

Not specified

\section{Health insurance}

MINSA

Formal workers (Essalud)

Army Force

Private

Not specified

\section{Place of death}

Health center

Home

Public/transit road

Not specified

\section{Altitude}

$<500$ masl

500 - 2999 masl

$>=3000$ masl

\section{Natural region}

Lima-Callao

Coast

Highlands

Jungle

\section{Poverty quintile ${ }^{b}$}

Quintile 5

Quintile 4

Quintile 3

Quintile 2

Quintile 1

\section{Non-communicable diseases}

Coronary disease

$$
\begin{array}{lllll}
10710 & (11.1) & 20404 & (10.8) & 9694 \\
20030 & (20.8) & 55840 & (29.6) & 35810
\end{array}
$$

$\begin{array}{lllll}48846 \quad(50.8) & 88604 & (47.0) & 39758\end{array}$

$28488 \quad(29.6) \quad 68822 \quad(36.5) \quad 40334 \quad$ (142)

$2225 \quad(2.3) \quad 5603 \quad(3.0) \quad 3378$

4745

(4.9) $\quad 8875$

(4.7) $\quad 4130$

11779

(12.3) $\quad 16574$

(8.8) $\quad 4795$

$\begin{array}{llllll}54523 & (56.7 & 109873 & (58.3) & 55350 & (102) \\ 34635 & (36.0) & 71865 & (38.1) & 37230 & (107) \\ 6681 & (7.0) & 6439 & (3.4) & -242 & (-4) \\ 244 & (0.3) & 301 & (0.2) & 57 & (23)\end{array}$

$55180 \quad(57.7) \quad 132542 \quad(70.5) \quad 77362$

$21025 \quad(22.0) \quad 31158 \quad(16.6) \quad 10133$

$\begin{array}{lllll}19376 & (20.3) \quad(13.0) \quad 49369 \quad(26)\end{array}$

$\begin{array}{llllll}30121 & (31.5) & 72633 & (38.6) & 42512 & (141) \\ 19880 & (20.8) & 51397 & (27.3) & 31517 & (159) \\ 34856 & (36.5) & 47491 & (25.3) & 12635 & (36) \\ 10724 & (11.2) & 16548 & (8.8) & 5824 & (54)\end{array}$

$\begin{array}{llllll}57854 & (60.5) & 129578 & (68.9) & 71724 & (124) \\ 17089 & (17.9) & 33499 & (17.8) & 16410 & (96) \\ 8719 & (9.1) & 12317 & (6.5) & 3598 & (41) \\ 6962 & (7.3) & 7681 & (4.1) & 719 & (10) \\ 4957 & (5.2) & 4994 & (2.7) & 37 & (1)\end{array}$

$7347 \quad$ (7.6) $21450 \quad(11.4) \quad 14103$




$\begin{array}{lcccccc}\text { Stroke } & 7214 & (7.5) & 9558 & (5.1) & 2344 & (32) \\ \text { Diabetes } & 5964 & (6.2) & 16437 & (8.7) & 10473 & (176) \\ \text { Hypertension } & 8006 & (8.3) & 24399 & (12.9) & 16393 & (205) \\ \text { Kidney failure } & 7698 & (8.0) & 11013 & (5.8) & 3315 & (43) \\ \text { Obesity } & 251 & (0.3) & 3676 & (2.0) & 3425 & (1365) \\ \text { basic cause of death } & & & & & & \\ \text { Respiratory infections }^{\mathrm{c}} & 29611 & (30.8) & 87069 & (46.2) & 57458 & (194) \\ \text { Others infections }^{\mathrm{d}} & 12375 & (12.9) & 19720 & (10.5) & 7345 & (59) \\ \text { Cardiovascular disease }^{\mathrm{e}} & 11135 & (11.6) & 23719 & (12.6) & 12584 & (113) \\ \text { Other non-communicable disease }^{\mathrm{f}} & 35566 & (37.0) & 47309 & (25.1) & 11743 & (33) \\ \text { External injuries }^{\mathrm{g}} & 5851 & (6.1) & 8322 & (4.4) & 2471 & (42) \\ \text { Not specified } & 1545 & (1.6) & 2339 & (1.2) & 794 & (51)\end{array}$

Source: National System of Deaths (SINADEF) March 01- December 31, 2019, and 2020. MINSA:Health Ministery. Masl: meters above sea level.

${ }^{\mathrm{b}}$ Poverty according to the National Institute of Statistics and Informatics from Peru (INEI-2020).

\section{General mortality in COVID19 first wave}

The epidemiological characteristics of pandemic first wave compared to 2019 showed a higher prevalence of males, older, college/technical instruction, attended in EsSalud hospitals (formal workers' Insurance), with the place of death in health centers, living in altitude less than 500 meters above sea level such as Lima-Callao, and poverty quintile five (less needy). (Table 1)

The accumulated mortality rate from COVID-19 first wave, according to SINADEF data in December 2020, was 25.1 deaths per 10000 inhabitants. However, official MINSA data underreported COVID deaths compared to the SINADEF database in 54\%. (Figure 1). The exact gap by the Peruvian region is in Supplementary Table 3 and 4.

Supplementary table 3. Mortality excess COVID-19 first wave in Peru.

\begin{tabular}{|c|c|c|c|c|c|c|}
\hline \multicolumn{3}{|c|}{ 2019-2020 compared mortality } & \multicolumn{4}{|c|}{2020 mortality } \\
\hline $\begin{array}{c}\text { Deaths } \\
2019\end{array}$ & $\begin{array}{c}\text { Deaths } \\
2020\end{array}$ & $\begin{array}{c}\text { Mortality } \\
\text { excess }\end{array}$ & $\begin{array}{c}\text { COVID- } \\
19\end{array}$ & $\begin{array}{c}\text { Confirme } \\
\text { d COVID- } \\
19^{b}\end{array}$ & $\begin{array}{c}\text { Suspected } \\
\text { COVID- } \\
19^{c}\end{array}$ & $\begin{array}{c}\text { Non } \\
\text { COVID- } \\
19^{\mathrm{d}}\end{array}$ \\
\hline
\end{tabular}




\begin{tabular}{lcccccccc} 
& $\mathrm{n}$ & $\mathrm{n}$ & $\Delta$ & $\%$ & $\mathrm{n}(\%)$ & $\mathrm{n}(\%)$ & $\mathrm{n}(\%)$ & $\mathrm{n}(\%)$ \\
\hline TOTAL & 96083 & 188478 & +92395 & $+96 \%$ & $81919(43)$ & $48903(26)$ & $33016(17)$ & $106559(57)$ \\
\hline Mar & 9818 & 9512 & -306 & $-3 \%$ & $85(1)$ & $2(0)$ & $83(1)$ & $9247(99)$ \\
Apr & 8880 & 12532 & +3652 & $+41 \%$ & $3776(30)$ & $1176(9)$ & $2600(21)$ & $8756(70)$ \\
May & 8938 & 24243 & +15305 & $+171 \%$ & $13128(54)$ & $5211(21)$ & $7917(33)$ & $11115(46)$ \\
Jun & 9332 & 25967 & +16635 & $+178 \%$ & $14735(57)$ & $8224(32)$ & $6511(25)$ & $11232(43)$ \\
Jul & 9918 & 35322 & +25404 & $+256 \%$ & $20176(57)$ & $12956(37)$ & $7220(20)$ & $15146(43)$ \\
Aug & 9959 & 26520 & +16561 & $+166 \%$ & $15070(57)$ & $10669(40)$ & $4401(17)$ & $11450(43)$ \\
Sep & 9713 & 16094 & +6381 & $+66 \%$ & $6865(43)$ & $4994(31)$ & $1871(12)$ & $9229(57)$ \\
Oct & 9980 & 13291 & +3311 & $+33 \%$ & $3220(24)$ & $2274(17)$ & $946(7)$ & $10071(76)$ \\
Nov & 9579 & 11915 & +2336 & $+24 \%$ & $2163(18)$ & $1527(13)$ & $636(5)$ & $9752(82)$ \\
Dec & 9966 & 13082 & +3116 & $+31 \%$ & $2701(21)$ & $1870(14)$ & $831(7)$ & $10381(79)$ \\
\hline
\end{tabular}

Source: National System of Deaths (SINADEF) March 01 to December 31, 2019 and 2020.

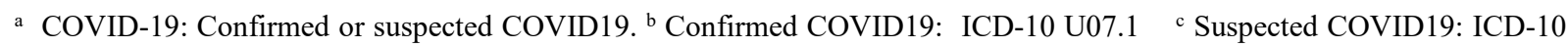
(U07.2 , B34.X or J97.X) or by written diagnosis ("COVID", "Coronavirus", "SARS CoV-2").

${ }^{\mathrm{d}}$ Non COVID-19: Confirmed or suspected absent COVID-19.

\section{Supplementary fig 1 . Monthly mortality comparative 2019 -2020 COVID-19 first wave}

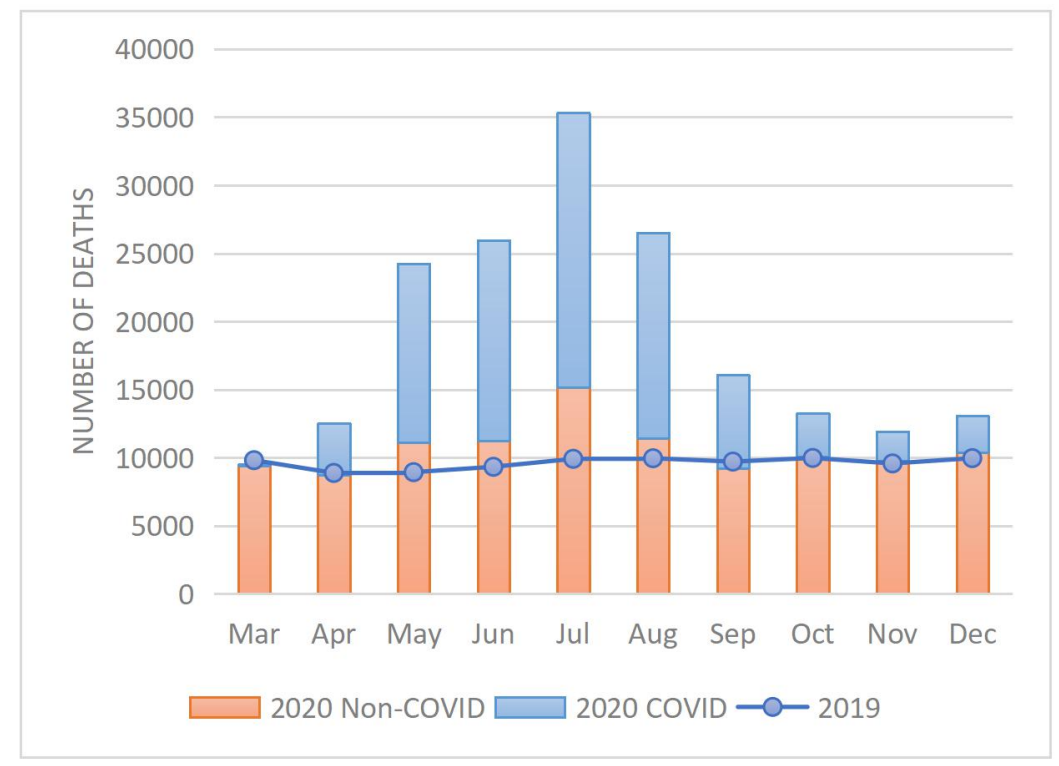

Source: National System of Deaths (SINADEF) March 01 to December 31, 2019 and 2020

COVID-19: Confirmed or suspected COVID19. Confirmed COVID19: ICD-10 U07.1 Suspected COVID19: ICD-10 (U07.2 , B34.X or J97.X) or by written diagnosis ("COVID", "Coronavirus", "SARS CoV-2"). Non COVID-19: Confirmed or suspected absent COVID-19

CHD: Coronary heart disease. DM: Diabetes mellitus HTN: Hypertension KF: Kidney failure. Obesity: OBE. 
Supplementary table 4. Underreport according to Peruvian region from March 01 to December 31, 2020.

\begin{tabular}{|c|c|c|c|c|c|c|}
\hline Región & Population & $\begin{array}{l}\text { Official } \\
\text { deaths }\end{array}$ & $\begin{array}{c}\text { SINADEF } \\
\text { deaths }\end{array}$ & $\begin{array}{c}\text { Official } \\
\text { deaths rate } x \\
10000 \text { inh }\end{array}$ & $\begin{array}{c}\text { SINADEF } \\
\text { deaths rate } \\
\text { x } 10000 \\
\text { inh } \\
\end{array}$ & $\begin{array}{l}\text { \% Under } \\
\text { report }\end{array}$ \\
\hline ** PERU ** & $32,625,948$ & 37724 & 81,924 & 11.56 & 25.11 & $54 \%$ \\
\hline Amazonas & 426806 & 254 & 346 & 5.95 & 8.11 & $27 \%$ \\
\hline Ancash & 1180638 & 1517 & 2867 & 12.85 & 24.28 & $47 \%$ \\
\hline Apurimac & 430736 & 159 & 381 & 3.69 & 8.85 & $58 \%$ \\
\hline Arequipa & 1497438 & 1630 & 4129 & 10.89 & 27.57 & $61 \%$ \\
\hline Ayacucho & 668213 & 378 & 571 & 5.66 & 8.55 & $34 \%$ \\
\hline Cajamarca & 1453711 & 611 & 1435 & 4.20 & 9.87 & $57 \%$ \\
\hline Callao & 1129854 & 1979 & 4660 & 17.52 & 41.24 & $58 \%$ \\
\hline Cusco & 1357075 & 533 & 1422 & 3.93 & 10.48 & $63 \%$ \\
\hline Huancavelica & 365317 & 147 & 453 & 4.02 & 12.40 & $68 \%$ \\
\hline Huánuco & 760267 & 494 & 1037 & 6.50 & 13.64 & $52 \%$ \\
\hline Ica & 975182 & 1794 & 3444 & 18.40 & 35.32 & $48 \%$ \\
\hline Junín & 1361467 & 969 & 2320 & 7.12 & 17.04 & $58 \%$ \\
\hline La Libertad & 2016771 & 2480 & 4319 & 12.30 & 21.42 & $43 \%$ \\
\hline Lambayeque & 1310785 & 1938 & 2856 & 14.79 & 21.79 & $32 \%$ \\
\hline Lima & 10628470 & 16791 & 38307 & 15.80 & 36.04 & $56 \%$ \\
\hline Loreto & 1027559 & 1008 & 1689 & 9.81 & 16.44 & $40 \%$ \\
\hline Madre de Dios & 173811 & 156 & 442 & 8.98 & 25.43 & $65 \%$ \\
\hline Moquegua & 192740 & 310 & 778 & 16.08 & 40.37 & $60 \%$ \\
\hline Pasco & 271904 & 138 & 312 & 5.08 & 11.47 & $56 \%$ \\
\hline Piura & 2047954 & 2227 & 5098 & 10.87 & 24.89 & $56 \%$ \\
\hline Puno & 1237997 & 403 & 1260 & 3.26 & 10.18 & $68 \%$ \\
\hline San Martín & 899648 & 797 & 1351 & 8.86 & 15.02 & $41 \%$ \\
\hline Tacna & 370974 & 270 & 602 & 7.28 & 16.23 & $55 \%$ \\
\hline Tumbes & 251521 & 346 & 645 & 13.76 & 25.64 & $46 \%$ \\
\hline Ucayali & 589110 & 395 & 1038 & 6.71 & 17.62 & $62 \%$ \\
\hline
\end{tabular}

Source: Official deaths from Ministery of health . SINADEF ( National system of deceased in Spanish). 
Figure 1. Underreport and mortality rates of official and SINADEF deaths from Peruvian regions during COVID-19 first wave

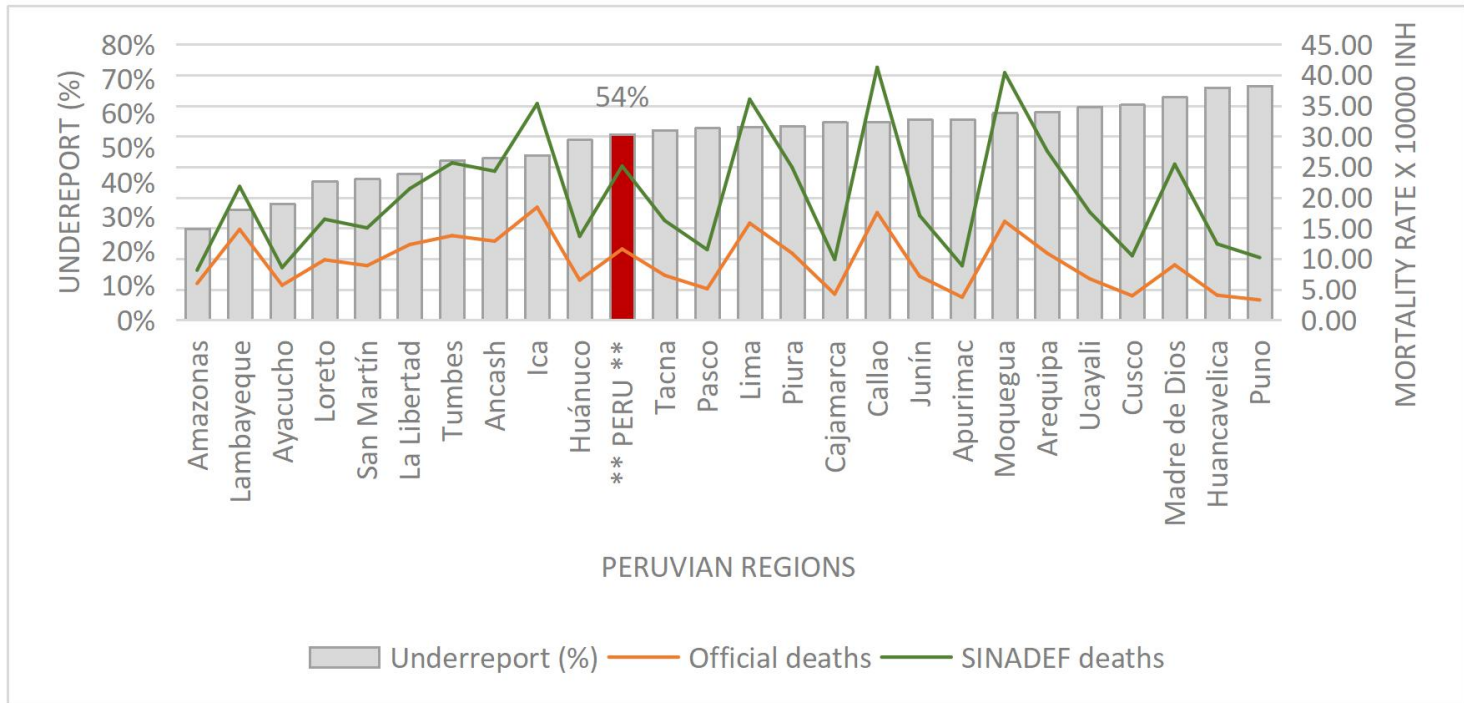

Source: National System of Deaths (SINADEF) and COVID-19 mortality MINSA, March 01- December 31, 2020.

According basic cause of death, the nation-wide cardiovascular mortality trend from 2017 to 2019 was between 11.5 to $12.0 \%$. In absolute values of 2020 , cardiovascular deaths increased in almost $100 \%$. (Supplementary table 5).

Supplementary table 5. Causes of mortality in Peruvian population 2017- 2020

\begin{tabular}{lcccc}
\hline & 2017 & 2018 & 2019 & 2020 \\
\hline General & 98977 & 112809 & 114946 & 207435 \\
Cardiovascular $^{\text {a }}$ & $11839(12.0)$ & $13833(12.3)$ & $13262(11.5)$ & $25937(12.5)$ \\
Other non-communicable $^{\mathbf{b}}$ & $38245(38.6)$ & $45912(40.7)$ & $42396(36.9)$ & $54958(26.5)$ \\
Respiratory infection $^{\mathbf{c}}$ & $28193(28.5)$ & $31784(28.2)$ & $35557(30.9)$ & $92622(44.7)$ \\
Other infections $^{\mathbf{d}}$ & $14050(14.2)$ & $13088(11.6)$ & $15100(13.1)$ & $21719(12.5)$ \\
External injuries $^{\mathbf{e}}$ & $6434(6.5)$ & $7593(6.7)$ & $7043(6.1)$ & $9782(4.7)$ \\
Not encoded $^{\mathrm{f}}$ & $216(0.2)$ & $599(0.5)$ & $1588(1.4)$ & $2417(1.2)$ \\
\hline
\end{tabular}

Source: National System of Deaths (SINADEF) 2017 -2020

c Respiratory infections: ICD-10 codes: J18 - j22, J80, and J96. ' ${ }^{\mathrm{d}}$ Others infections: ICD-10 codes: A00 -A99, B00-B99, J00-J15 and G00-G03,

e Cardiovascular disease: ICD-10 codes: $\mathrm{I} 20$ to I25 and I60 to I69. ${ }^{\mathrm{f}}$ Other non-communicable diseases. ICD10 chapters: C, D, E, F, G, H, I00-I19, I26-I59; I70-I99, K, L, M, N, O, P, Q, J30 - J99, except J80 and J96 . ' External injuries: ICD-10 chapters: S, T, V, W, X, Y. Not encoded: Includes cardiorespiratory arrest, decomposition without the possibility of knowing the cause, among others 


\section{Mortality according to COVID19}

During the pandemic first wave, 57\% of deaths were due to non-COVID disease. These deaths were mainly women, older, with less instruction, care in the public health system (MINSA), deaths occurred at home, and more significant poverty (quintile 3, 2, and 1). (Table 2).

Table 2. Clinical and epidemiological characteristics of deaths in 2020 according to COVID disease during the first wave

\begin{tabular}{lccccc}
\hline & \multicolumn{2}{c}{ Non-COVID19 } & COVID19 & p-value \\
& $\mathrm{n}$ & $\%$ & $\mathrm{~N}$ & $\%$ & \\
\hline General & 106556 & $100 \%$ & 81918 & 100
\end{tabular}

Sex

$\begin{array}{llllll}\text { Female } & 49098 & 46.1 \% & 25091 & 30.6 & \\ \text { Male } & 57458 & 53.9 \% & 56827 & 69.4 & <0.001\end{array}$

Age group

$<18$ y

$18-59$ y

$>60 \mathrm{y}$

Instruction degree

Illiterate

Elementary School

High School

College/Technical

Not specified

Health insurance

Public (MINSA)

Formal workers (Essalud)

Army Force

Private

Not specified

Place of death

$\begin{array}{ccccc}5395 & 5.1 \% & 415 & 0.5 & <0.001 \\ 22635 & 21.2 \% & 23169 & 28.3 & \\ 78494 & 73.7 \% & 58318 & 71.2\end{array}$

$\begin{array}{lllll}19953 & 18.7 \% & 5053 & 6.2 & <0.001 \\ 33599 & 31.5 \% & 10740 & 13.1 & \\ 25768 & 24.2 \% & 17121 & 20.9 & \\ 13265 & 12.4 \% & 7139 & 8.7 & \\ 13974 & 13.1 \% & 41866 & 51.1 & \end{array}$

$\begin{array}{lllll}52854 & 49.6 \% & 35750 & 43.6 & <0.001 \\ 35390 & 33.2 \% & 33432 & 40.8 & \\ 2342 & 2.2 \% & 3261 & 4.0 & \\ 5616 & 5.3 \% & 3259 & 4.0 & \\ 10357 & 9.7 \% & 6217 & 7.6 & \end{array}$


Health center

Home

Public/transit road

Not specified

\section{Altitude}

$<500$ masl

500 - 2999 masl

$>=3000$ masl

Natural region

Lima-Callao

Coast

Highlands

Jungle

\section{Poverty quintile ${ }^{b}$}

Quintile 5

Quintile 4

Quintile 3

Quintile 2

Quintile 1

\section{Non-communicable diseases}

Coronary disease$$
\text { No }
$$$$
\text { Sí }
$$

Stroke

$$
\begin{aligned}
& \text { No } \\
& \text { Sí }
\end{aligned}
$$

Diabetes

No

Sí

Hypertension

No

$\begin{array}{llll}37022 & 34.7 \% & 72851 & 88.9 \\ 64250 & 60.3 \% & 7615 & 9.3 \\ 5107 & 4.8 \% & 1332 & 1.6 \\ 180 & 0.2 \% & 121 & 0.1\end{array}$

$68145 \quad 64.1 \%$

64397

$78.8<0.001$

20295

$19.1 \%$

10863

13.3

17878

$16.8 \%$

6491

7.9

$\begin{array}{llccl}38808 & 36.5 \% & 33825 & 41.4 & <0.001 \\ 24712 & 23.2 \% & 26685 & 32.6 & \\ 32904 & 30.9 \% & 14587 & 17.8 & \\ 9894 & 9.3 \% & 6654 & 8.1 & \end{array}$

$\begin{array}{llccl}68976 & 64.9 \% & 60602 & 74.1 & <0.001 \\ 18973 & 17.8 \% & 14526 & 17.8 & \\ 8519 & 8.0 \% & 3798 & 4.6 & \\ 5968 & 5.6 \% & 1713 & 2.1 & \\ 3882 & 3.7 \% & 1112 & 1.4\end{array}$

85682

80.4

81346

$99.3<0.001$

20877

19.6

573

0.7

98112

92.1

80808

$98.6<0.001$

8447

7.9

1111

1.4

96776

90.8

75265

$91.9<0.001$

9783

9.2

6654

8.1 


$\begin{array}{lccccc}\text { No } & 89261 & 83.8 & 74818 & 91.3 & <0.001 \\ \text { Sí } & 17298 & 16.2 & 7101 & 8.7 & \end{array}$

Kidney failure

$\begin{array}{lccccc}\text { No } & 98472 & 92,4 & 78993 & 96.4 & <0.001 \\ \text { Sí } & 8087 & 7.6 & 2926 & 3.6 & \end{array}$

Obesity

$\begin{array}{lccccc}\text { No } & 106047 & 99.5 & 78755 & 96.1 & <0.001 \\ \text { Sí } & 512 & 0.5 \% & 3164 & 3.9 & \end{array}$

Source: National System of Deaths (SINADEF) March 01- December 31, 2019, a, and 2020. MINSA:Health Ministery. Masl: meters above sea level.

COVID-19: Confirmed or suspected COVID19. Confirmed COVID19: ICD-10 U07.1 Suspected COVID19: ICD-10 (U07.2 , B34.X or J97.X) or by written diagnosis ("COVID", "Coronavirus", "SARS CoV-2"). Non COVID-19: Confirmed o suspected COVID-19 absent. ${ }^{b}$ Poverty according to the National Institute of Statistics and Informatics from Peru (INEI2020).

A $97.3 \%$ of subjects with coronary disease died due non-COVID19 causes, $88.4 \%$ with stroke, a $73.4 \%$ with kidney failure, $70.9 \%$ in hypertension, $59.5 \%$ in diabetes mellitus, and $13.9 \%$ in obesity. (Table 3 and Figure 2)

Table 3. General mortality according to cardiometabolic diseases during the first wave

\begin{tabular}{|c|c|c|c|c|c|c|}
\hline $\begin{array}{l}\text { Non comunicable } \\
\text { diseases }\end{array}$ & Cases / Total & $\begin{array}{c}\text { General } \\
\text { mortality } \\
\text { Prevalence } \\
\text { (IC95\%) }\end{array}$ & $\begin{array}{c}\text { Crude } \\
\text { análisis } \\
\text { PR } \\
\text { (CI95\%) }\end{array}$ & $\begin{array}{c}\text { p- } \\
\text { value }\end{array}$ & $\begin{array}{c}\text { Adjusted } \\
\text { análisis } \\
\text { PR (CI95\%) }\end{array}$ & $\begin{array}{c}\text { p- } \\
\text { value }\end{array}$ \\
\hline \multicolumn{7}{|l|}{$\begin{array}{l}\text { Positive COVID19 } \\
\text { deaths } \\
\text { Coronary } \\
\text { disease }\end{array}$} \\
\hline No & $\begin{array}{l}81346 / \\
167028\end{array}$ & $\begin{array}{l}48.7(48.4- \\
48.9)\end{array}$ & 1.00 & & 1.00 & \\
\hline Sí & $5737 / 21450$ & $2.7(2.62-2.73)$ & $\begin{array}{l}0.05(0.05- \\
0.06\end{array}$ & $<0.001$ & $\begin{array}{l}0.13(0.12- \\
0.14)\end{array}$ & $<0.001$ \\
\hline \multicolumn{7}{|l|}{ Stroke } \\
\hline No & $\begin{array}{c}80808 \\
/ 178920\end{array}$ & $\begin{array}{l}45.2(44.9- \\
\quad 45.4)\end{array}$ & 1.00 & & 1.00 & \\
\hline Sí & $1111 / 9558$ & $\begin{array}{l}11.6(11.0- \\
12.3)\end{array}$ & $\begin{array}{l}0.25(0.24- \\
0.27)\end{array}$ & $<0.001$ & $\begin{array}{c}0.30(0.29- \\
0.32)\end{array}$ & $<0.001$ \\
\hline \multicolumn{7}{|l|}{ Diabetes } \\
\hline No & $\begin{array}{l}75265 \\
/ 172041\end{array}$ & $\begin{array}{l}43.8(43.5- \\
\quad 43.9)\end{array}$ & 1.00 & & 1.00 & \\
\hline Sí & 6654 / 16437 & $\begin{array}{l}40.5(39.7- \\
41.2)\end{array}$ & $\begin{array}{l}0.93(0.91- \\
0.94)\end{array}$ & $<0.001$ & $\begin{array}{l}0.95(0.94- \\
0.96)\end{array}$ & $<0.001$ \\
\hline Hypertension & & & & & & \\
\hline
\end{tabular}




\begin{tabular}{ccccccc}
\hline No & $74818 /$ & $45.6(39.7-$ & 1.00 & & 1.00 \\
& 164079 & $41.2)$ & & & \\
Sí & $7101 / 24399$ & $29.1(28.5-$ & $0.64(0.63-$ & $<0.001$ & $0.99(0.97-$ & 0.087 \\
& & $29.7)$ & $0.65)$ & & $1.001)$ & $0.001)$
\end{tabular}

Kidney failure

\begin{tabular}{|c|c|c|c|c|c|c|}
\hline No & $\begin{array}{l}78993 / \\
177465\end{array}$ & $\begin{array}{c}44.5(44.3- \\
44.7)\end{array}$ & 1.00 & & 1.00 & \\
\hline$a^{\prime}$ & $2926 / 11013$ & $\begin{array}{c}26.6(25.7- \\
27.4)\end{array}$ & $\begin{array}{c}0.60(0.58- \\
0.62)\end{array}$ & $<0.001$ & $\begin{array}{c}0.58(0.57- \\
0.60)\end{array}$ & $<0.001$ \\
\hline
\end{tabular}

Obesity

\begin{tabular}{|c|c|c|c|c|c|c|}
\hline No & $\begin{array}{l}78755 / \\
184802\end{array}$ & $\begin{array}{c}42.6(42.4- \\
42.8)\end{array}$ & 1.00 & & 1.00 & \\
\hline Sí & $3164 / 3676$ & $\begin{array}{c}86.1(84.9- \\
87.2)\end{array}$ & $\begin{array}{c}2.01(1.99- \\
2.05)\end{array}$ & $<0.001$ & $\begin{array}{c}1.37(1.35- \\
1.39)\end{array}$ & $<0.001$ \\
\hline
\end{tabular}

\section{Negative COVID19 deaths disease \\ Coronary}

$\begin{array}{cc} & 85682 / \\ \text { No } & 167028 \\ \text { Sí } & 20877 / \\ & 21450\end{array}$

Stroke

$\begin{array}{cc} & 98112 \\ \text { No } & / 178920 \\ & 8447 / 9558\end{array}$

\section{Diabetes}

$\begin{array}{cc}\text { No } & 96776 \\ & / 172041 \\ \text { Sí } & 9783 / 16437\end{array}$

\section{Hypertension}

$\begin{array}{lc} & 89261 / \\ \text { No } & 164079 \\ \text { Sí } & 17298 / \\ & 24399\end{array}$

Kidney failure

No

$$
\text { Sí }
$$

$$
\begin{aligned}
& 98472 / \\
& 177465
\end{aligned}
$$$$
8087 / 11013
$$

$$
\begin{gathered}
51.3(51.1- \\
51.5) \\
97.3(97.1- \\
97.5)
\end{gathered}
$$

$$
1.00
$$$$
\begin{gathered}
1.90(1.89- \\
1.91)
\end{gathered}<0.001
$$

$$
1.00
$$

$\begin{gathered}1.29(1.28- \\ 1.30)\end{gathered}<0.001$

$$
\begin{array}{ccccc}
54.8(54.6- & 1.00 & & 1.00 & \\
55.1) & & & 1.51(1.49- & <0.001 \\
88.4(87.7- & 1.61(1.60- & <0.001 & 1.52) & \\
89.0) & 1.63) & & 1.04)
\end{array}
$$

$$
\begin{gathered}
56.3(56.0- \\
56.5) \\
59.5(58.8- \\
60.3)
\end{gathered}
$$

$$
1.00
$$$$
1.00
$$$$
1.06(1.05-
$$$$
\text { 1.08) }
$$

$<0.001$

$$
\begin{array}{ll}
1.07) & <0.001
\end{array}
$$

$$
\begin{gathered}
54.4(54.2- \\
54.6) \\
70.9(70.3- \\
71.4)
\end{gathered}
$$

$$
1.00
$$$$
1.00
$$$$
1.30(1.29-<0.001
$$

$1.00(0.99-$

1.01)

0.984

1.32)

$$
\begin{gathered}
55.5(55.2- \\
55.7) \\
73.4(72.6- \\
74.3)
\end{gathered}
$$

1.00

$1.32(1.31-<0.001$
$1.34)$

$\begin{array}{cl}1.39(1.37- & <0.001 \\ 1.41) & \end{array}$

\section{Obesity}

\begin{tabular}{lcccccc} 
No & $106047 /$ & $57.4(57.2-$ & 1.00 & & 1.00 & \\
& 184802 & $57.6)$ & $0.24(0.22-$ & $<0.001$ & $0.37(0.34-$ & $<0.001$ \\
Sí & $512 / 3676$ & $13.9(12.8-$ & $0.24)$ & $0.39)$ & \\
\hline
\end{tabular}


FIg 2. Non-communicable disease mortality comparative 2019-2020 COVID-19 first wave

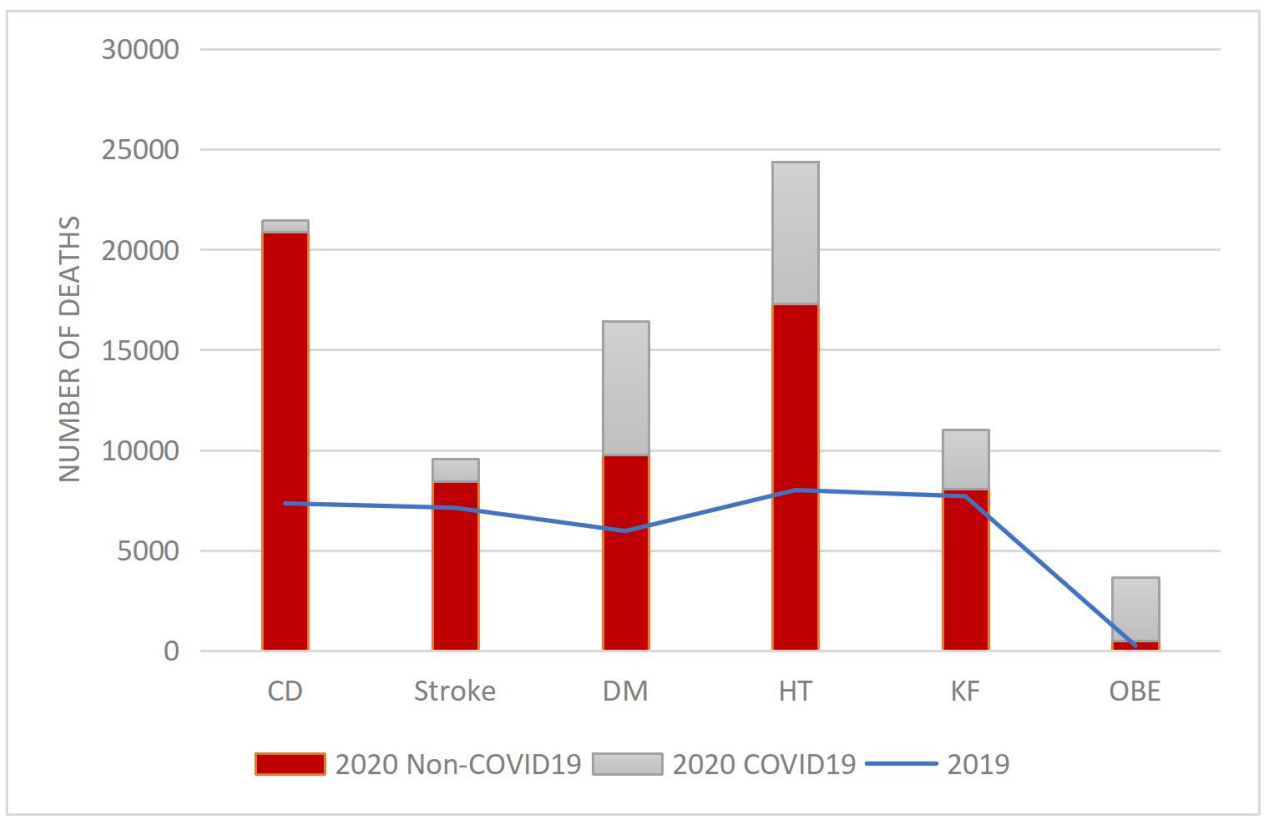

Source: National System of Deaths (SINADEF) March 01 to December 31, 2019, and 2020

COVID-19: Confirmed or suspected COVID19. Confirmed COVID19: ICD-10 U07.1 Suspected COVID19: ICD-10 (U07.2 , B34.X or J97.X) or by written diagnosis ("COVID", "Coronavirus", "SARS CoV-2"). Non COVID-19: Confirmed or suspected absent COVID-19

CD: Coronary disease. DM: Diabetes mellitus HT: Hypertension KF: Kidney failure. Obesity: OBE.

Adjusted to epidemiological characteristics, general mortality due non-COVID causes was $51 \%$ more frequent in stroke (PR 1.51; CI95\% 1.49-1.52; $<<0.001$ ); 29\% in coronary disease (PR 1.29 CI95\% 1.28-1.30;p<0.001), and 5\% in diabetes (PR 1.06; CI95\% 1.051.08; $<0.001$ ). General mortality due COVID cause was $37 \%$ more frequent in obesity (PR 1.37; CI95\%1.35-1.-39; $\mathrm{p}<0.001$ ). (Table 3). Full multivariate regression is shown in Supplementary table 6 .

Supplementary table 6. Full Multivariate regression of associated factors of non-COVID mortality,

\begin{tabular}{|c|c|c|c|c|c|c|c|c|}
\hline & \multicolumn{2}{|c|}{ Non-COVID } & \multicolumn{2}{|c|}{ COVID } & \multirow[t]{2}{*}{ Crude analysis } & \multirow[t]{2}{*}{$\begin{array}{c}\mathrm{p}- \\
\text { value }\end{array}$} & \multirow[t]{2}{*}{$\begin{array}{l}\text { Adjusted } \\
\text { analysis }\end{array}$} & \multirow[t]{2}{*}{ p-value } \\
\hline & $\mathrm{n}$ & $\%$ & $\mathrm{~N}$ & $\%$ & & & & \\
\hline General & 106556 & $100 \%$ & 81918 & 100 & & & & \\
\hline \multicolumn{9}{|l|}{ Sex } \\
\hline Female & 49098 & $46.1 \%$ & 25091 & 30.6 & 1.00 & & 1.00 & \\
\hline Male & 57458 & $53.9 \%$ & 56827 & 69.4 & $0.75(0.75-0.77)$ & $<0.001$ & $0.85(0.85-0.86)$ & $<0.001$ \\
\hline
\end{tabular}

Age group 


$\begin{array}{lcccccccc}<18 \text { y } & 5395 & 5.1 \% & 415 & 0.5 & 1.00 & & 1.00 & \\ 18-59 y & 22635 & 21.2 \% & 23169 & 28.3 & 0.53(0.51-0.55) & <0.001 & 0.61(0.60-0.62) & <0.001 \\ >60 y & 78494 & 73.7 \% & 58318 & 71.2 & 0.62(0.60-0.64) & <0.001 & 0.59(0.58-0.60) & <0.001\end{array}$

\section{Instruction degree}

$\begin{array}{lcccccccc}\text { Illiterate } & 19953 & 18.7 \% & 5053 & 6.2 & 1.00 & & 1.00 & \\ \text { Elementary School } & 33599 & 31.5 \% & 10740 & 13.1 & 0.95(0.93-0.97) & <0.001 & 0.98(0.97-0.99) & <0.001 \\ \text { High School } & 25768 & 24.2 \% & 17121 & 20.9 & 0.75(0.74-0.77) & <0.001 & 0.91(0.90-0.92) & <0.001 \\ \text { College/Technical } & 13265 & 12.4 \% & 7139 & 8.7 & 0.81(0.80-0.83) & <0.001 & \begin{array}{c}0.99(0.98- \\ 1.001)\end{array} & 0.094 \\ \text { Not specified } & 13974 & 13.1 \% & 41866 & 51.1 & 0.31(0.30-0.32) & <0.001 & 0.54(0.53-0.55) & <0.001\end{array}$

\section{Health insurance}

$\begin{array}{lcccccccc}\text { Public (MINSA) } & 52854 & 49.6 \% & 35750 & 43.6 & 1.00 & & 1.00 & \\ \begin{array}{l}\text { Formal workers } \\ \text { (Essalud) }\end{array} & 35390 & 33.2 \% & 33432 & 40.8 & 0.86(0.85-0.87) & <0.001 & 1.01(0.99-1.02) & 0.088 \\ \text { Army Force } & 2342 & 2.2 \% & 3261 & 4.0 & 0.70(0.67-0.73) & <0.001 & 0.92(0.90-0.95) & <0.001 \\ \text { Private } & 5616 & 5.3 \% & 3259 & 4.0 & 1.06(1.03-1.09) & <0.001 & 1.15(1.13-1.16) & <0.001 \\ \text { Not specified } & 10357 & 9.7 \% & 6217 & 7.6 & 1.05(0.03-1.07) & <0.001 & 1.06(1.05-1.07) & <0.001\end{array}$

\section{Place of death}

$\begin{array}{lcccccccc}\text { Health center } & 37022 & 34.7 \% & 72851 & 88.9 & 1.00 & & 1.00 & \\ \text { Home } & 64250 & 60.3 \% & 7615 & 9.3 & 2.65(2.63-2.67) & <0.001 & 1.97(1.96-1.99) & <0.001 \\ \text { Public/transit road } & 5107 & 4.8 \% & 1332 & 1.6 & 2.35(2.31-2.39) & <0.001 & 1.86(1.83-1.89) & <0.001 \\ & 180 & 0.2 \% & 121 & 0.1 & 1.77(1.61-1.95) & <0.001 & 1.56(1.43-1.71) & <0.001\end{array}$

Altitude

\begin{tabular}{|c|c|c|c|c|c|c|c|c|}
\hline$<500$ masl & 68145 & $64.1 \%$ & 64397 & 78.8 & 1.00 & & 1.00 & \\
\hline $500-2999$ masl & 20295 & $19.1 \%$ & 10863 & 13.3 & $1.27(1.25-1.29)$ & $<0.001$ & $1.08(1.06-1.10)$ & $<0.001$ \\
\hline$>=3000$ masl & 17878 & $16.8 \%$ & 6491 & 7.9 & $1.42(1.40-1.45)$ & $<0.001$ & $1.11(1.08-1.13)$ & $<0.001$ \\
\hline
\end{tabular}

\section{Natural region}

$\begin{array}{lcccccccr}\text { Lima-Callao } & 38808 & 36.5 \% & 33825 & 41.4 & 1.00 & & 1.00 & \\ \text { Coast } & 24712 & 23.2 \% & 26685 & 32.6 & 0.90(089 .-0.91) & <0.001 & 0.89(0.88-0.90) & <0.001 \\ \text { Highlands } & 32904 & 30.9 \% & 14587 & 17.8 & 1.29(1.28-1.31) & <0.001 & \begin{array}{c}0.98(0.96- \\ 1.004)\end{array} & 0.123 \\ \text { Jungle } & 9894 & 9.3 \% & 6654 & 8.1 & 1.12(1.10-1.14) & <0.001 & 0.99(0.98-1.01) & 0.684\end{array}$

\section{Poverty quintil ${ }^{b}$}




$\begin{array}{lcccccccc}\text { Quintil 5 } & 68976 & 64.9 \% & 60602 & 74.1 & 1.00 & & 1.00 & \\ \text { Quintil 4 } & 18973 & 17.8 \% & 14526 & 17.8 & 1.06(1.05-1.07) & <0.001 & 0.97(0.96-0.98) & <0.001 \\ \text { Quintil 3 } & 8519 & 8.0 \% & 3798 & 4.6 & 1.30(1.28-1.31) & <0.001 & 1.04(1.03-1.05) & <0.001 \\ \text { Quintil 2 } & 5968 & 5.6 \% & 1713 & 2.1 & 1.46(1.44-1.48) & <0.001 & 1.04(1.02-1.05) & <0.001 \\ \text { Quintil 1 } & 3882 & 3.7 \% & 1112 & 1.4 & 1.46(1.44-1.48) & <0.001 & 1.05(1.03-1.06) & <0.001\end{array}$

Non comunicable

diseases

Coronary disease

\begin{tabular}{lcccccccl} 
No & 85682 & 80.4 & 81346 & 99.3 & 1.00 & \multicolumn{2}{l}{1.00} \\
Sí & 20877 & 19.6 & 573 & 0.7 & $1.90(1.89-1.91)$ & $<0.001$ & $1.29(1.28-1.30)$ & $<0.001$
\end{tabular}

Stroke

\begin{tabular}{lllllcccl} 
No & 98112 & 92.1 & 80808 & 98.6 & 1.00 & \multicolumn{2}{l}{1.00} \\
Sí & 8447 & 7.9 & 1111 & 1.4 & $1.61(1.60-1.63)$ & $<0.001$ & $1.51(1.49-1.52)$ & $<0.001$
\end{tabular}

Diabetes

\begin{tabular}{lrrrrcccll} 
No & 96776 & 90.8 & 75265 & 91.9 & 1.00 & \multicolumn{2}{l}{1.00} \\
Sí & 9783 & 9.2 & 6654 & 8.1 & $1.05(1.04-1.07)$ & $<0.001$ & $1.06(1.05-1.08)$ & $<0.001$
\end{tabular}

Hypertension

\begin{tabular}{lrrrrcccl} 
No & 89261 & 83.8 & 74818 & 91.3 & 1.00 & \multicolumn{2}{l}{1.00} \\
Sí & 17298 & 16.2 & 7101 & 8.7 & $1.30(1.29-1.32)$ & $<0.001$ & $1.00(0.99-1.01)$ & 0.984
\end{tabular}

Kidney failure

$\begin{array}{ccccccccc}\text { No } & 98472 & 92,4 & 78993 & 96.4 & 1.00 & & 1.00 & \\ \text { Sí } & 8087 & 7.6 & 2926 & 3.6 & 1.32(1.31-1.34) & <0.001 & 1.39(1.37-1.41) & <0.001\end{array}$

Obesity

\begin{tabular}{ccccccccc} 
No & 106047 & 99.5 & 78755 & 96.1 & 1.00 & & 1.00 & \\
Sí & 512 & $0.5 \%$ & 3164 & 3.9 & $0.24(0.22-0.26)$ & $<0.001$ & $0.37(0.34-0.39)$ & $<0.001$ \\
\hline
\end{tabular}

\section{Cardiovascular mortality in non-COVID19.}

The prevalence of mortality due cardiovascular cause in the first wave was $21.5 \%$ in nonCOVID deaths and $0.95 \%$ in COVID deaths. In the same way, cardiovascular mortality in subjects with hypertension was $35.1 \%$, in diabetes was $16.8 \%$, and in obesity was $5.1 \%$. Adjusted to epidemiological characteristics, cardiovascular mortality was 13.2 times more frequent in non-COVID-19; 2.3 times in subjects with hypertension (PR 2.31; CI95\% 2.24- 
2.37; $<0.001$ ), 31\% more in subjects with obesity (PR 1.31; CI95\% 1.14-1.52;p<0.001) and 3\% more in subjects with diabetes mellitus (PR 1.03; CI95\% 0.99-1.07; p<0.090). (Table $4)$.

Table 4. Cardiovascular mortality in Peruvian population during the first wave

\begin{tabular}{ccccccc}
\hline & $\begin{array}{c}\text { Cases } \\
\text { /Total }\end{array}$ & $\begin{array}{c}\text { Cardivoascular } \\
\text { mortality } \\
\text { Prevalence \% } \\
\text { (IC95\%) }\end{array}$ & $\begin{array}{c}\text { Crude } \\
\text { analysis } \\
\text { PR (I95\%) }\end{array}$ & $\begin{array}{c}\text { P- } \\
\text { value }\end{array}$ & $\begin{array}{c}\text { Adjusted } \\
\text { analysis } \\
\text { PR (I95\%) }\end{array}$ & $\begin{array}{c}\text { P- } \\
\text { value }\end{array}$ \\
\hline COVID19 & & & & & & \\
Yes & $782 /$ & $0.95(0.89-1.02)$ & 1.00 & & 1.00 & \\
& 81919 & & & & 13.29 & \\
Not & $22937 /$ & $21.5(21.3-21.8)$ & $\begin{array}{c}22.5(20.9- \\
\text { (I9.2) }\end{array}$ & $<0.001$ & $(12.32-$ & $<0.001$ \\
& 106559 & & & & $14.34)$ &
\end{tabular}

Sex

$\begin{array}{lcccccl}\text { Female } & 10603 / & 14.3(14.0-14.5) & 1.00 & & 1.00 & \\ & 74189 & & 0.80(0.78 & <0.001 & 1.05(1.03- & \\ \text { Male } & 13116 & 11.5(11.3-11.7) & -0.82) & & 1.08) & <0.001\end{array}$

Age group

$\begin{array}{lc}<18 \text { y } & 258 / \\ & 5810 \\ 18-59 y & 3652 / \\ & 45804 \\ >60 y & 19807 / \\ & 136812\end{array}$

$\begin{array}{ccccc}4.4(3.9-5.0) & 1.00 & & 1.00 & \\ & 1.79(1.58- & <0.001 & 2.31(2.03- & <0.001 \\ 7.9(7.7-8.2) & 2.03) & & 2.63) & \\ & 3.26(2.88- & <0.001 & 2.72(2.40- & <0.001 \\ 14.5(14.3-14.7) & 3.68) & & 3.09) & \end{array}$

Instruction degree

\begin{tabular}{|c|c|c|c|c|c|c|}
\hline Illiterate & $\begin{array}{l}3471 / \\
25006\end{array}$ & $13.9(13.4-14.3)$ & 1.00 & & 1.00 & \\
\hline $\begin{array}{l}\text { Elementary } \\
\text { School }\end{array}$ & $\begin{array}{l}8929 / \\
44339\end{array}$ & $20.1(19.8-20.5)$ & $\begin{array}{l}1.45(1.39- \\
1.51)\end{array}$ & $<0.001$ & $\begin{array}{c}1.08(1.04- \\
1.12)\end{array}$ & $<0.001$ \\
\hline High School & $\begin{array}{l}6244 / \\
42889\end{array}$ & $14.6(14.2-14.9)$ & $\begin{array}{c}1.04(1.01- \\
1.09)\end{array}$ & 0.024 & $\begin{array}{c}1.04(1.002- \\
1.09)\end{array}$ & 0.040 \\
\hline College/Technical & $\begin{array}{l}2906 / \\
20404\end{array}$ & $14.2(13.8-14.7)$ & $\begin{array}{c}1.03(0.97- \\
1.08)\end{array}$ & 0.306 & $\begin{array}{l}0.95(0.90- \\
1.01)\end{array}$ & 0.111 \\
\hline Not specified & $\begin{array}{l}2169 / \\
55840\end{array}$ & $3.9(3.72-4.05)$ & $\begin{array}{l}0.27(0.26- \\
0.30)\end{array}$ & $<0.001$ & $\begin{array}{l}0.86(0.82- \\
0.92)\end{array}$ & $<0.001$ \\
\hline \multicolumn{7}{|l|}{ Health insurance } \\
\hline MINSA & $\begin{array}{c}10648 / \\
88648\end{array}$ & $12.0(11.8-12.2)$ & 1.00 & & 1.00 & \\
\hline $\begin{array}{l}\text { Formal workers } \\
\text { (Essalud) }\end{array}$ & $\begin{array}{l}8565 \\
/ 68822\end{array}$ & $12.5(8.1-9.6)$ & $\begin{array}{l}1.03(1.01- \\
1.07)\end{array}$ & 0.016 & $\begin{array}{l}0.99(0.96- \\
1.02)\end{array}$ & 0.591 \\
\hline Army Force & $\begin{array}{l}494 / \\
5603\end{array}$ & $8.8(14.1-15.6)$ & $\begin{array}{l}0.73(0.67- \\
0.80)\end{array}$ & $<0.001$ & $\begin{array}{l}0.97(0.89- \\
1.07)\end{array}$ & 0.642 \\
\hline Private & $\begin{array}{l}1316 / \\
8875\end{array}$ & $14.8(15.7-16.8)$ & $\begin{array}{c}1.23(1.16- \\
1.30)\end{array}$ & $<0.001$ & $\begin{array}{c}1.11(1.04- \\
1.17)\end{array}$ & 0.001 \\
\hline Not specified & $\begin{array}{l}2696 / \\
16574\end{array}$ & $16.3(15.7-16.8)$ & $\begin{array}{c}1.35(1.29- \\
1.41)\end{array}$ & $<0.001$ & $\begin{array}{c}1.14(1.09- \\
1.19)\end{array}$ & $<0.001$ \\
\hline
\end{tabular}


Place of death

\begin{tabular}{|c|c|c|c|c|c|c|}
\hline Health center & $\begin{array}{c}4578 / \\
109873\end{array}$ & $4.2(4.1-4.3)$ & 1.00 & & 1.00 & \\
\hline Home & $\begin{array}{l}18482 / \\
71865\end{array}$ & $25.7(25.4-26.0)$ & $\begin{array}{c}6.17(5.97- \\
6.37)\end{array}$ & $<0.001$ & $\begin{array}{c}2.10(2.02- \\
2.18)\end{array}$ & $<0.001$ \\
\hline ablic/transit road & $\begin{array}{l}640 / \\
6439\end{array}$ & $9.9(9.2-10.7)$ & $\begin{array}{c}2.38(2.19- \\
2.59)\end{array}$ & $<0.001$ & $\begin{array}{c}1.22(1.12- \\
1.33)\end{array}$ & $<0.001$ \\
\hline t specified & $19 / 301$ & $6.3(3.8-9.7)$ & $\begin{array}{c}1.51(0.96- \\
2.37)\end{array}$ & 0.071 & $\begin{array}{c}1.09(0.69- \\
1.72)\end{array}$ & 0.686 \\
\hline
\end{tabular}

Altitude

$\begin{array}{lc}<500 \text { masl } & 18014 / \\ & 132542 \\ 500-2999 \text { masl } & 3458 \\ & / 31158 \\ >=3000 \text { masl } & 2217 / \\ & 24369\end{array}$

$\begin{array}{ccc}13.6(13.4-13.8) & 1.00 \\ & 0.81(0.78- & \\ 11.1(10.8-11.5) & 0.84) & <0.001 \\ & 0.67(0.64- & \end{array}$

1.00

$9.1(8.73-9.45)$

$\begin{array}{ll}0.67(0.64- & <0.001 \\ 0.70) & \end{array}$

$\begin{array}{cc}1.02(0.94- & 0.527 \\ 1.12) & \end{array}$

$0.74(0.67-<0.001$

Natural región

$\begin{array}{lc}\text { Lima-Callao } & 10278 / \\ & 72633 \\ \text { Coast } & 7271 \\ & 151397 \\ \text { Highlands } & 4852 / \\ & 47491 \\ \text { Jungle } & 1288 / \\ & 16548\end{array}$

$\begin{array}{ccc}14.2(13.8-14.4) & 1.00 \\ 14.2(13.8-14.5) & 0.99(0.97- & 0.986 \\ & 1.03) & \\ 10.2(9.9-10.5) & 0.72(0.69- & <0.001 \\ & 0.74) & \\ 7.8(7.4-8.2) & 0.55(0.52- & <0.001 \\ & 0.58) & \end{array}$

1.00

$1.04(1.01$ -

$1.07)$

0.007

$0.75(0.68-<0.001$

$0.82)$

$0.60(0.56-<0.001$

Poverty quintile ${ }^{b}$

Quintile 5

$16038 /$ 129578

Quintil 4

33499

Quintil 3

1558 /

12317

Quintil 2

794 /

7681

Quintil 1

$521 /$

4994

$\begin{array}{ccc}12.4(12.2-12.6) & 1.00 \\ & 1.15(1.11- & <0.001 \\ 14.3(13.9-14.6) & 1.19) & \\ & 1.02(0.97- & 0.413 \\ 12.7(12.1-13.2) & 1.07) & \\ & 0.83(0.78- & <0.001 \\ 10.3(9.66-11.03) & 0.90) & \\ & 0.84(0.77- & <0.001\end{array}$

1.00

$1.14(1.10-$

$1.18)$

$0.65)$

$0.97(0.92-1-$

03)

$0.90(0.83-\quad 0.009$

$0.97)$

$0.95(0.86-$

1.04)

$<0.001$

0.307

$0.92)$

0.294

Cardiometabolic

diseases

Diabetes

$\begin{array}{ll} & 20959 \\ \text { No } & 17204 \\ & 2760 / \\ \text { Sí } & 16437\end{array}$

$12.2(12.0-12.3)$

1.00

1.00

16.8 (16.2-17-4)

$\begin{gathered}1.37(1.32- \\ 1.43)\end{gathered}<0.001$

$1.03(0.99$ -

1.07)

0.090

Hypertension

$\begin{array}{lcccccl}\text { No } & 15151 / & 9.2(9.09-9.37) & 1.00 & & 1.00 & \\ & 164079 & & & & & \\ & 8568 & 35.1(34.5-35.7) & -3.90 & <0.001 & 2.31(2.24-2- & <0.001 \\ \text { Sí } & / 24399 & & -3.90 & 37) & \end{array}$

Obesity

No

$23533 /$

1.00

1.00 


\begin{tabular}{ccccccc}
\hline \multirow{2}{*}{ Sí } & \multirow{2}{*}{$186 / 3676$} & $5.1(4.4-5.8)$ & $\begin{array}{c}0.40(0.34 \\
-0.45)\end{array}$ & $<0.001$ & $\begin{array}{c}1.31(1.14- \\
1.52)\end{array}$ & $<0.001$ \\
\hline
\end{tabular}

\section{DISCUSSION}

The data obtained show a very high mortality excess in Peru during the COVID-19 pandemic first wave near $100 \%$ compared to the 2019 rate. Three out of five were unrelated to COVID19. The associated cardiometabolic diseases in COVID-19 deaths were obesity, while nonCOVID-19 deaths were stroke, coronary disease, and diabetes mellitus.

Excess deaths during the COVID-19 pandemic have been reported in most countries; however, the health policies for each country have led to mortality not directly related to the infection. [21] A comparative study of global excess mortality until March 2021 ranks Peru in the first place of excess death with more than 355 deaths per 100,000 inhabitants, while Mexico, Ecuador, Bolivia as well Eastern European countries and South Africa, maintain excess mortality between 200 and 300 deaths per 100,000 inhabitants [1].

In the first quarter of 2020, Wuhan showed an increase of $56 \%$ compared to 2019 . This increase was due to COVID-19 in 8 times. But also cardiovascular disease and DM increased in $29 \%$ and $83 \%$ respectively [22]. In the United Kingdom until June 2020, 94\% of acute cardiovascular deaths were in people without COVID-19, showing an absolute excess compared to the previous year of $8 \%$, mostly occurring at home with an increase of $35 \%$. The most common conditions were stroke $36 \%$, acute coronary disease $25 \%$, and heart failure 23\%. [23]. In Denmark, there was no excess mortality in 2020 compared to 2019; however, the home mortality rate increased by $4 \%$, being ore frequent in those with heart failure, peripheral arterial disease, and DM [24]. In Latin America, reports on excess death are scarce, and the frequency of its components is uncertain [6].

A multifactorial approach can explain this excess death due to non-COVID deaths. For example, deterioration in the support network of the primary health care level, the timely work of the health team in the care of the health emergency was hampered by the paralysis of the outpatient clinic and the conversion of general hospitals to exclusive ones for COVID-19 and lack of recognition of warning signs to go to emergency services $[25,26]$. A survey by the World Health Organization (WHO) carried out in 105 countries between May and June 2020 described that $90 \%$ of the surveyed countries partially or wholly interrupted at least 1 of the 25 essential health services, being the most frequent immunization services, noncommunicable diseases, and family planning services [27]. 
In Non-COVID patients, the results show a slight association between diabetes and hypertension with mortality. But this relationship is non-precious because the basic cause of death is coronary disease and stroke. In addition, we observed a progressive trend since 15 years in cardiometabolic mortality, and we expected high mortality percentages in light of these extreme confinement measures.[10]

Some authors classify death from COVID 19 as a cardiovascular and non-infectious cause. This rating is due to thrombosis, and anticoagulant treatment is promoted in patients with severe disease.[28] Less than 1\% of deaths with COVID were presented as the basic cause of cardiovascular death. The high risk of contagion and the public policy of cremation and early burial limited the autopsy of these patients. [29]

Public health importance

The extreme restrictive measures applied in the same way without differentiating geographical risk, indirectly increased Non-COVID death. Coronary heart disease or stroke was the typical way of death for a large part of this population with diabetes, hypertension, or obesity. It is not clear, the true impact of non-covid mortality on the population. We must be describe mortality characteristics and factors as a first step and then to start improvement plans,. [30,31]

Likewise, two epidemiological mortality phenotypes identified during the first wave. The first who died with COVID-19 were males, with death in health establishments and Quintile 5 of poverty. A second one corresponded to non-COVID-19 person, mainly women, older, with less education, $60 \%$ of deaths occurred at home and greatest poverty (quintile 3, 2 and 1).

The underreport of 54\% was officially recognized in April 2021. Suspected cases were included, thus reducing the information gap. The data reaffirmed that we maintain the place with the highest mortality. However, many countries also have information gaps. Comparisons should be made considering similar calculation methodologies and the type of death report, and the diagnostic method used for COVID. [32].

\section{Limitations and strengths}

The limitations in this investigation were the quality of completion of death certificates by physicians, including lack of documentation of the basic cause of death and the lack of coding [33]. Also, serological tests during the first months of the pandemic delayed a molecular diagnosis and the confirmed COVID-19 code. The reference to determine excess mortality was 2019; other authors use the expected mortality taken from the last 5 years. 
Among the strengths, we were able to determine the basic cause of death in all the data, since about $50 \%$ of it does not have an ICD10 coding. We also add poverty level, altitude and natural region of each deceased, important confounding variables in the mortality analysis.

\section{Conclusion}

During the first wave of COVID-19 in Peru, three out of five were unrelated to COVID-19. The associated cardiometabolic diseases in COVID-19 deaths were obesity, while nonCOVID-19 deaths were stroke, coronary disease, and diabetes mellitus. Historical, social shortcomings, such as the universal health insurance gap, the inequity of basic services, labor informality, and an expanding epidemiological transition, have triggered this excessive mortality [34].

\section{Contributors}

SNS was el author of the idea and design of the investigation. SNS and MYA were the authors of the statistics and interpretation of results. SNS and MYA participated in the research and article preparation and approved the final article.

\section{Acknowledgment}

The authors appreciate the review of Julio Leey, MD, Division of Endocrinology, Diabetes and Metabolism, University of Florida, Gainesville, Florida, in preparing the manuscript.

\section{REFERENCES}

[1] The Economist. Tracking covid-19 excess deaths across countries. Econ 2021. https://www.economist.com/graphic-detail/coronavirus-excess-deaths-tracker (accessed March 11, 2021).

[2] Adjwanou V, Alam N, Alkerna L, Asiki G, Bawah A, Beguy D. Measuring excess mortality during the COVID-19 pandemic in low- and lower-middle income countries: the need for mobile phone surveys. SocArXiv 2020. https://doi.org/https://doi.org/10.31235/osf.io/4bu3q. 
[3] Magnani C, Azzolina D, Gallo E, Ferrante D, Gregori D. How large was the mortality increase directly and indirectly caused by the COVID-19 epidemic? An analysis on allcauses mortality data in Italy. Int J Environ Res Public Health 2020;17:3452. https://doi.org/10.3390/ijerph17103452.

[4] Woolf SH, Chapman DA, Sabo RT, Weinberger DM, Hill L, Taylor DSDH. Excess Deaths from COVID-19 and Other Causes, March-July 2020. JAMA - J Am Med Assoc 2020;324:1562-4. https://doi.org/10.1001/jama.2020.19545.

[5] Nogueirai PJ, De Araújo Nobre M, Nicola PJ, Furtado C, Vaz Carneiro A. Excess mortality estimation during the COVID-19 pandemic: Preliminary data from Portugal. Acta Med Port 2020;33:376-83. https://doi.org/10.20344/amp.13928.

[6] Lima E, Vilela E, Peralta A, Rocha MG, Queiroz BL, Gonzaga MR. Exploring Excess of Deaths in the Context of Covid Pandemic in Selected Countries of Latin America. OSF Prepr 2020. https://doi.org/https://doi.org/10.31219/osf.io/xhkp4.

[7] Grillo-Rojas PF, Romero-Onofre R. Estimate of the excess of the total deaths reported in 2020 versus the reported deaths from COVID-19 (SARS-CoV2) in Peru during the months of March, April and May 2020. Rev Fac Med Hum 2020;20:646-50. https://doi.org/https://doi.org/10.25176/RFMH.v20i4.3220.

[8] Taylor L. Covid-19: Why Peru suffers from one of the highest excess death rates in the world. BMJ 2021:372:n611. https://doi.org/https://doi.org/10.1136/bmj.n611.

[9] Bernabé-Ortiz A, Carrillo-Larco RM. La transición epidemiológica en el Perú: análisis de los registros de mortalidad del 2003 al 2016. ACTA MEDICA Peru 2020;37. https://doi.org/10.35663/amp.2020.373.1550.

[10] Atamari-Anahui N, Ccorahua-Rios M, Taype-Rondan A, Mejia CR. Mortalidad atribuida a diabetes mellitus registrada en el Ministerio de Salud de Perú, 2005-2014. Rev Panam Salud Pública 2018;42:e50. https://doi.org/10.26633/rpsp.2018.50.

[11] Ministerio de Salud. Directiva Administrativa 216-MINSA/OGTI-V.01: Directiva Administrativa que establece el Procedimiento para la Certificación de las Defunciones, aprobada mediante Resolución Ministerial 280-2016/MINSA Perú 2016:38. ftp://ftp2.minsa.gob.pe/descargas/ogei/SINADEF/RM_280-2016-

DIRECTIVA_DEFUNCIONES.pdf (accessed January 15, 2021).

[12] Ministerio de Salud. Información de Fallecidos del Sistema Informático Nacional de Defunciones - SINADEF - $\quad$ [Ministerio de Salud] 2021. https://www.datosabiertos.gob.pe/dataset/información-de-fallecidos-del-sistemainformático-nacional-de-defunciones-sinadef-ministerio (accessed January 15, 2021). 
[13] Ministerio de Salud. Directiva Sanitaria para la Vigilancia Epidemiológica de la Enfermedad por Coronavirus (COVID-19) en el Perú. 2020:1-40. https://www.dge.gob.pe/portalnuevo/wp-content/uploads/2021/01/Directiva-devigilancia-de-COVID-19.pdf (accessed January 15, 2021).

[14] Instituto Nacional de Estadística e Informática. Perú: Estimaciones y Proyecciones de Población por Departamento, Provincia y Distrito, 2018-2020. Boletín Espec ํ26 2020:110.

https://www.inei.gob.pe/media/MenuRecursivo/publicaciones_digitales/Est/Lib1715/libro. pdf (accessed January 15, 2021).

[15] Instituto Nacional de Estadística e Informática. Compendio Estadístico del Perú 2013:1523.

https://www.inei.gob.pe/media/MenuRecursivo/publicaciones_digitales/Est/Lib1097/libro. pdf.

[16] Instituto Nacional de Estadística e Informática. Mapa de pobreza monetaria provincial y distrital 2018. 2020:303

pp. https://www.inei.gob.pe/media/MenuRecursivo/publicaciones_digitales/Est/Lib1718/Libr o.pdf.

[17] Ministerio de Salud - Dirección General de Tecnologías de la Información. Guía técnica para el correcto llenado del certificado de defunción 2018:69 pp. http://bvs.minsa.gob.pe/local/MINSA/4459.pdf.

[18] Organización Panamericana de la Salud. Lineamientos básicos para el análisis de la mortalidad 2017:145. https://iris.paho.org/bitstream/handle/10665.2/34492/9789275319819spa.pdf?sequence=7 (accessed January 15, 2021).

[19] Dirección General de Epidemiología - Ministerio de Salud. Metodología para el análisis de situación de salud local 2015:97. http://bvs.minsa.gob.pe/local/MINSA/3399.pdf (accessed January 15, 2021).

[20] Ministerio de Salud. Fallecidos por COVID-19 - [Ministerio de Salud - MINSA] 2021. https://www.datosabiertos.gob.pe/dataset/fallecidos-por-covid-19-ministerio-de-saludminsa (accessed January 15, 2021).

[21] Karlinsky A, Kobak D. Tracking excess mortality across countries during the covid-19 pandemic with the world mortality dataset. Elife 2021;10. https://doi.org/10.7554/ELIFE.69336.

[22] Liu J, Zhang L, Yan Y, Zhou Y, Yin P, Qi J, et al. Excess mortality in Wuhan city and 
other parts of China during the three months of the covid-19 outbreak: Findings from nationwide mortality registries. BMJ 2021;372. https://doi.org/10.1136/bmj.n415.

[23] Wu J, Mamas MA, Mohamed MO, Kwok CS, Roebuck C, Humberstone B, et al. Place and causes of acute cardiovascular mortality during the COVID-19 pandemic. Heart 2021;107:113-9. https://doi.org/10.1136/heartjnl-2020-317912.

[24] Butt JH, Fosbøl EL, Gerds TA, Andersson C, Kragholm K, Biering-Sørensen T, et al. Allcause mortality and location of death in patients with established cardiovascular disease before, during, and after the COVID-19 lockdown: a Danish Nationwide Cohort Study. Eur Heart J 2021. https://doi.org/10.1093/eurheartj/ehab028.

[25] Siriaco M. La estrategia olvidada: ¿qué pasó con los centros de atención primaria? Salud Con Lupa 2020. https:/saludconlupa.com/noticias/la-estrategia-olvidada-que-paso-conlos-centros-de-atencion-primaria/ (accessed February 12, 2021).

[26] Pesantes MA, Lazo-Porras M, Cárdenas MK, Diez-Canseco F, Tanaka-Zafra JH, CarrilloLarco RM, et al. Los retos del cuidado de las personas con diabetes durante el estado de emergencia nacional por la COVID-19 en Lima, Perú: recomendaciones para la atención primaria. Rev Peru Med Exp Salud Publica 2020;37:541-6. https://doi.org/10.17843/rpmesp.2020.373.5980.

[27] World Health Organization. Pulse survey on continuity of essential health services during the COVID-19 pandemic: interim report, 27 August 2020 2020:21. https://www.who.int/publications/i/item/WHO-2019-nCoV-EHS_continuity-survey2020.1 (accessed February 12, 2021).

[28] Wichmann D, Sperhake J-P, Lütgehetmann M, Steurer S, Edler C, Heinemann A, et al. Autopsy Findings and Venous Thromboembolism in Patients With COVID-19. Https://DoiOrg/107326/M20-2003 2020;173:268-77. https://doi.org/10.7326/M20-2003.

[29] Elezkurtaj S, Greuel S, Ihlow J, Michaelis EG, Bischoff P, Kunze CA, et al. Causes of death and comorbidities in hospitalized patients with COVID-19. Sci Reports 2021111 2021;11:1-9. https://doi.org/10.1038/s41598-021-82862-5.

[30] Banerjee A, Chen S, Pasea L, Lai AG, Katsoulis M, Denaxas S, et al. Excess deaths in people with cardiovascular diseases during the COVID-19 pandemic. Eur J Prev Cardiol 2021. https://doi.org/10.1093/EURJPC/ZWAA155.

[31] Rao GH. Cardiometabolic Diseases: A Global Perspective. J Cardiol Cardiovasc Ther 2018;12:1-6. https://doi.org/10.19080/jocct.2018.12.555834.

[32] Lau H, Khosrawipour T, Kocbach P, Ichii H, Bania J, Khosrawipour V. Evaluating the massive underreporting and undertesting of COVID-19 cases in multiple global 
epicenters.

Pulmonology

$2021 ; 27: 110-5$.

https://doi.org/10.1016/J.PULMOE.2020.05.015.

[33] Soto Cáceres Cabanillas R, Fernandez- Mogollón JL, Daniel VV. Calidad de llenado del certificado de defunción en dos hospitales de Chiclayo,Perú, 2016. Rev Exp En Med Del Hosp Reg Lambayeque 2019;5. https://doi.org/10.37065/rem.v5i2.344.

[34] Mesa de Concertación para la Lucha contra la Pobreza. Informe Nacional sobre el impacto del COVID.19 en ls dimensiones económica, social y en salud en el Perú. n.d.:88. https://www.mesadeconcertacion.org.pe/storage/documentos/2020-08-17/informe-saludmclcp-2020-7-de-julio.pdf (accessed February 12, 2021). 